\title{
Explicit a priori bounds on transfer operator eigenvalues
}

\author{
Oscar F. Bandtlow and Oliver Jenkinson
}

\begin{abstract}
We provide explicit bounds on the eigenvalues of transfer operators defined in terms of holomorphic data.
\end{abstract}

Linear operators of the form $\mathcal{L} f=\sum_{i \in \mathcal{I}} w_{i} \cdot f \circ T_{i}$, so-called transfer operators (see e.g. Bal, Rue1, Rue2]), arise in a number of problems in dynamical systems. If the $T_{i}$ are inverse branches of an expanding map $T$, and the weight functions $w_{i}$ are positive, the spectrum of $\mathcal{L}$ has well-known interpretations in terms of the exponential mixing rate of an invariant Gibbs measure (see $[\mathbf{B a l}]$ ). Applications also arise when the $w_{i}$ are real-valued (e.g. [CCR, JMS, Pol]) or complex-valued (e.g. [Dol, [PS]).

In this article we suppose that $T_{i}$ and $w_{i}$ are analytic functions of $d$ variables, for each $i$ in some countable 1 index set $\mathcal{I}$. Under suitable hypotheses on $T_{i}$ and $w_{i}$ the transfer operator $\mathcal{L}$ defines a compact operator on Hardy space $H^{2}(B)$, and we can give completely explicit bounds on its eigenvalue sequence $\left\{\lambda_{n}(\mathcal{L})\right\}_{n=1}^{\infty}$ :

Theorem 1. Suppose there is a complex Euclidean ball $B \subset \mathbb{C}^{d}$ such that each $w_{i}: B \rightarrow \mathbb{C}$ is holomorphic with $\sum_{i \in \mathcal{I}} \sup _{z \in B}\left|w_{i}(z)\right|<\infty$, and each $T_{i}: B \rightarrow B$ is holomorphic with $\cup_{i \in \mathcal{I}} T_{i}(B)$ contained in the ball concentric with $B$ whose radius is $r<1$ times that of $B$.

Then $\mathcal{L}: H^{2}(B) \rightarrow H^{2}(B)$ is compact and

$$
\left|\lambda_{n}(\mathcal{L})\right|<\frac{W \sqrt{d}}{r^{d}\left(1-r^{2}\right)^{d / 2}} n^{(d-1) /(2 d)} r^{\frac{d}{d+1}(d !)^{1 / d} n^{1 / d}} \quad \text { for all } n \geq 1,
$$

where $W:=\sup _{z \in B} \sum_{i \in \mathcal{I}}\left|w_{i}(z)\right|$.

If $d=1$ then

$$
\left|\lambda_{n}(\mathcal{L})\right| \leq \frac{W}{\sqrt{1-r^{2}}} r^{(n-1) / 2} \quad \text { for all } n \geq 1 .
$$

Remark 2.

(i) An estimate of the form $\left|\lambda_{n}(\mathcal{L})\right| \leq C \theta^{n^{1 / d}}$ for some (undefined) constants $C>0, \theta \in(0,1)$ is asserted, either implicitly or explicitly, in the work of several authors (e.g. [FR, Fri, GLZ]); the novelty here is that careful derivation of this bound renders explicit the constants $C, \theta$.

(ii) Using different techniques, the bound $\left|\lambda_{n}(\mathcal{L})\right| \leq C \theta^{n^{1 / d}}$ can also be established in the case where $B$ is an arbitrary open subset of $\mathbb{C}^{d}$ (see $[\mathbf{B J}]$ ), though here our expressions for $C, \theta$ are more complicated.

Example 3. If $\mathcal{L} f(z)=\sum_{n=1}^{\infty}\left(\frac{1}{n+z}\right)^{2} f\left(\frac{1}{n+z}\right)$ (the Perron-Frobenius operator for the Gauss map $x \mapsto 1 / x(\bmod 1)$, cf. May $), B \subset \mathbb{C}$ may be chosen as the open disc of radius $3 / 2$

\footnotetext{
${ }^{1}$ Subsequent results are new even when $\mathcal{I}$ is finite, but it is convenient to also allow countably infinite $\mathcal{I}$.

${ }^{2}$ Precisely, $\left\{\lambda_{n}(\mathcal{L})\right\}_{n=1}^{\infty}$ denotes the sequence of all eigenvalues of $\mathcal{L}$ counting algebraic multiplicities and ordered by decreasing modulus, with the usual convention (see e.g. [Pie, 3.2.20]) that distinct eigenvalues with the same modulus can be written in any order.
} 
centred at the point 1 . In this case $W=\sup _{z \in B} \sum_{n=1}^{\infty}|n+z|^{-2}=\sum_{n=1}^{\infty}(n-1 / 2)^{-2}=\pi^{2} / 2$ and $r=2 / 3$, so (2) yields

$$
\left|\lambda_{n}(\mathcal{L})\right| \leq \frac{3 \pi^{2}}{2 \sqrt{5}}(2 / 3)^{(n-1) / 2} \quad \text { for all } n \geq 1 .
$$

Notation 4. For an open ball $D \subset \mathbb{C}^{d}$, let $H^{\infty}(D)$ denote the Banach space consisting of all bounded holomorphic $\mathbb{C}$-valued functions on $D$, with norm $\|f\|_{H^{\infty}(D)}:=\sup _{z \in D}|f(z)|$.

Hardy space $H^{2}(D)$ (see $\left[\mathbf{K r a}\right.$, Ch. 8.3]) is the $L^{2}(\partial D, \sigma)$-closure of the set of those $f \in H^{\infty}(D)$ which extend continuously to the boundary $\partial D$, where $\sigma$ denotes $(2 d-1)$ dimensional Lebesgue measure on $\partial D$, normalised so that $\sigma(\partial D)=1$. In particular, $H^{2}(D)$ is a Hilbert subspace of $L^{2}(\partial D, \sigma)$ with each element $f \in H^{2}(D)$ having a natural holomorphic extension to $D$ (see [Kra, Ch. 1.5]).

In the sequel, no generality is lost by taking $B$ in the statement of Theorem 1 to be the unit ball $B_{1}$, and the smaller concentric ball to be $B_{r}$, the ball of radius $r$ centred at 0 .

If $L: X_{1} \rightarrow X_{2}$ is a continuous operator between Banach spaces then for $k \geq 1$, its $k$-th approximation number $a_{k}(L)$ is defined as

$$
a_{k}(L)=\inf \left\{\|L-K\| \mid K: X_{1} \rightarrow X_{2} \text { linear and continuous with } \operatorname{rank}(K)<k\right\} .
$$

The proof of Theorem 1 hinges on the following two lemmas.

Lemma 5. If $J: H^{2}\left(B_{1}\right) \hookrightarrow H^{\infty}\left(B_{r}\right)$ denotes the canonical embedding, then $J$ and $\mathcal{L}$ are compact and for all $n \geq 1$

$$
\left|\lambda_{n}(\mathcal{L})\right| \leq W \prod_{k=1}^{n} a_{k}(J)^{1 / n}
$$

Proof. If $f \in H^{2}\left(B_{1}\right)$ and $z \in B_{r}$ then $|f(z)| \leq(2 /(1-r))^{d / 2}$ by [Rud, Thm. 7.2.5], so $\left\{f \mid\|f\|_{H^{2}\left(B_{1}\right)} \leq 1\right\}$ is a normal family in $H^{\infty}\left(B_{r}\right)$, hence relatively compact in $H^{\infty}\left(B_{r}\right)$ by Montel's Theorem (see [Nar, Ch. 1, Prop. 6]), thus $J$ is compact.

Next observe that if $f \in H^{\infty}\left(B_{1}\right)$ then $f \in H^{2}\left(B_{1}\right)$ by [Rud, Thm. 5.6.8] and the canonical embedding $\hat{J}: H^{\infty}\left(B_{1}\right) \hookrightarrow H^{2}\left(B_{1}\right)$ is continuous of norm 1 , because $\sigma\left(\partial B_{1}\right)=1$. We claim that $\hat{\mathcal{L}} f:=\sum_{i \in \mathcal{I}} w_{i} \cdot f \circ T_{i}$ defines a continuous operator $\hat{\mathcal{L}}: H^{\infty}\left(B_{r}\right) \rightarrow H^{\infty}\left(B_{1}\right)$. To see this, fix $f \in H^{\infty}\left(B_{r}\right)$ and note that $w_{i} \cdot f \circ T_{i} \in H^{\infty}\left(B_{1}\right)$ with $\left\|w_{i} \cdot f \circ T_{i}\right\|_{H^{\infty}\left(B_{1}\right)} \leq$ $\left\|w_{i}\right\|_{H^{\infty}\left(B_{1}\right)}\|f\|_{H^{\infty}\left(B_{r}\right)}$ for every $i \in \mathcal{I}$. But since $\|\hat{\mathcal{L}} f\|_{H^{\infty}\left(B_{1}\right)} \leq \sum_{i \in \mathcal{I}}\left\|w_{i}\right\|_{H^{\infty}\left(B_{1}\right)}\|f\|_{H^{\infty}\left(B_{r}\right)}$ and $\sum_{i \in \mathcal{I}}\left\|w_{i}\right\|_{H^{\infty}\left(B_{1}\right)}<\infty$ by hypothesis, we conclude that $\hat{\mathcal{L}} f \in H^{\infty}\left(B_{1}\right)$ and that $\hat{\mathcal{L}}$ is continuous. Now $\left|f\left(T_{i}(z)\right)\right| \leq\|f\|_{H^{\infty}\left(B_{r}\right)}$ for every $z \in B_{1}, i \in \mathcal{I}$, so $\|\hat{\mathcal{L}} f\|_{H^{\infty}\left(B_{1}\right)}=$ $\sup _{z \in B_{1}}|(\hat{\mathcal{L}} f)(z)| \leq \sup _{z \in B_{1}} \sum_{i \in \mathcal{I}}\left|w_{i}(z)\right|\left|f\left(T_{i}(z)\right)\right| \leq W\|f\|_{H^{\infty}\left(B_{r}\right)}$, and hence $\|\hat{\mathcal{L}}\| \leq W$. Now clearly $\mathcal{L}=\hat{J} \hat{\mathcal{L}} J$, so $\mathcal{L}$ is compact, and

$$
a_{k}(\mathcal{L}) \leq\|\hat{J} \hat{\mathcal{L}}\| a_{k}(J) \leq W a_{k}(J) \text { for all } k \geq 1,
$$

since in general $a_{k}\left(L_{1} L_{2}\right) \leq\left\|L_{1}\right\| a_{k}\left(L_{2}\right)$ whenever $L_{1}$ and $L_{2}$ are bounded operators between Banach spaces (see [Pie, 2.2]). Moreover, since $\mathcal{L}$ is a compact operator on Hilbert space, Weyl's inequality (see [Pie, 3.5.1], Wey]) asserts that $\prod_{k=1}^{n}\left|\lambda_{k}(\mathcal{L})\right| \leq \prod_{k=1}^{n} a_{k}(\mathcal{L})$ for all $n \geq 1$. Together with (4) this yields (3), because $\left|\lambda_{n}(\mathcal{L})\right| \leq \prod_{k=1}^{n}\left|\lambda_{k}(\mathcal{L})\right|^{1 / n}$.

Lemma 6. If $h_{d}(k):=\left(\begin{array}{c}k+d \\ d\end{array}\right)$ then for all $n \geq 1$,

$$
a_{n}(J)^{2} \leq \sum_{l=k}^{\infty} h_{d-1}(l) r^{2 l} \quad \text { where } k \geq 0 \text { is such that } h_{d}(k-1)<n \leq h_{d}(k) .
$$


Proof. $H^{2}\left(B_{1}\right)$ has reproducing kernel $K(z, \zeta)=\left(1-(z, \zeta)_{\mathbb{C}^{d}}\right)^{-d}($ see $[\mathbf{K r a}$, Thm. 1.5.5] 3 ), where $(\cdot, \cdot)_{\mathbb{C}^{d}}$ denotes the Euclidean inner product, and $K(z, \zeta)=\sum_{n=1}^{\infty} p_{n}(z) \overline{p_{n}(\zeta)}$ whenever $\left\{p_{n}\right\}_{n=1}^{\infty}$ is an orthonormal basis for $H^{2}\left(B_{1}\right)$, the series converging pointwise for every $(z, \zeta) \in B_{1} \times B_{1}$ (see [Hal, p. 19]).

Define $J_{n}: H^{2}\left(B_{1}\right) \rightarrow H^{\infty}\left(B_{r}\right)$ by $J_{n} f=\sum_{k=1}^{n-1}\left(f, p_{k}\right) p_{k}$. If $z \in B_{r}$ then

$$
\begin{aligned}
\left|J f(z)-J_{n} f(z)\right|^{2}=\mid f(z) & -\left.J_{n} f(z)\right|^{2}=\left|\sum_{k=n}^{\infty}\left(f, p_{k}\right) p_{k}(z)\right|^{2} \\
\leq & \sum_{k=n}^{\infty}\left|\left(f, p_{k}\right)\right|^{2} \sum_{k=n}^{\infty}\left|p_{k}(z)\right|^{2} \leq\|f\|_{H^{2}\left(B_{1}\right)}^{2}\left(K(z, z)-\sum_{k=1}^{n-1}\left|p_{k}(z)\right|^{2}\right),
\end{aligned}
$$

so

$$
a_{n}(J)^{2} \leq \sup _{z \in B_{r}}\left(K(z, z)-\sum_{k=1}^{n-1}\left|p_{k}(z)\right|^{2}\right) .
$$

If $n=1$ then $k=0$, in which case (5) follows from (6) since $\sum_{l=0}^{\infty} h_{d-1}(l) r^{2 l}=\left(1-r^{2}\right)^{-d}$. Now define the orthonormal basis $\left\{p_{\underline{n}} \mid \underline{n} \in \mathbb{N}_{0}^{d}\right\}$ by (cf. [Rud, Prop. 1.4.8, 1.4.9])

$$
p_{\underline{n}}(z)=K_{\underline{n}} z^{\underline{n}} \quad\left(\underline{n} \in \mathbb{N}_{0}^{d}\right),
$$

where $K_{\underline{n}}=\sqrt{\frac{(|\underline{n}|+d-1) !}{(d-1) ! \underline{n} !}}, \underline{n}=\left(n_{1}, \ldots, n_{d}\right), z^{\underline{n}}=z_{1}^{n_{1}} \cdots z_{d}^{n_{d}}, \underline{n} !=n_{1} ! \cdots n_{d} !,|\underline{n}|=n_{1}+\cdots+n_{d}$.

If $n \geq 2$ then there are $\left(\begin{array}{c}k+d-1 \\ d\end{array}\right)$ multinomials of degree less than or equal to $k-1$, so

$$
a_{n}(J)^{2} \leq \sup _{z \in B_{r}}\left(K(z, z)-\sum_{|\underline{n}| \leq k-1}\left|p_{\underline{n}}(z)\right|^{2}\right)=\sup _{z \in B_{r}} \sum_{l=k}^{\infty} \sum_{|\underline{n}|=l}\left|p_{\underline{n}}(z)\right|^{2} \leq \sum_{l=k}^{\infty} \frac{(l+d-1) !}{(d-1) ! l !} r^{2 l}
$$

for all $n>\left(\begin{array}{c}k+d-1 \\ d\end{array}\right)$, because $\sum_{|\underline{n}|=l} \frac{1}{\underline{n} !}\left|z^{\underline{n}}\right|^{2} \leq \frac{1}{l !} r^{2 l}$ for $z \in B_{r}$ by the multinomial theorem.

Proof of Theorem 1. By Lemma[5]it suffices to bound the geometric means $\left(\prod_{k=1}^{n} a_{k}\right)^{1 / n}$, where $a_{k}:=a_{k}(J)$. From Lemma 6 it follows that

$$
a_{n}^{2} \leq \tilde{\alpha}_{n} \frac{r^{2 \tilde{\beta}_{n}}}{\left(1-r^{2}\right)^{d}} \quad \text { for all } n \geq 1
$$

where

$$
\begin{aligned}
& \tilde{\alpha}_{n}:=h_{d-1}(k) \quad \text { for } h_{d}(k-1)<n \leq h_{d}(k), \\
& \tilde{\beta}_{n}:=k
\end{aligned}
$$

because

$\sum_{l=k}^{\infty} h_{d-1}(l) r^{2 l}=h_{d-1}(k) r^{2 k} \sum_{l=0}^{\infty} \frac{h_{d-1}(l+k)}{h_{d-1}(k)} r^{2 l} \leq h_{d-1}(k) r^{2 k} \sum_{l=0}^{\infty} h_{d-1}(l) r^{2 l}=h_{d-1}(k) \frac{r^{2 k}}{\left(1-r^{2}\right)^{d}}$.

Combining (7) with Lemma 5 gives, for all $n \geq 1$,

$$
\left|\lambda_{n}(\mathcal{L})\right| \leq W \alpha_{n} \frac{r^{\beta_{n}}}{\left(1-r^{2}\right)^{d / 2}},
$$

where

$$
\alpha_{n}:=\prod_{l=1}^{n} \tilde{\alpha}_{l}^{1 /(2 n)}, \quad \beta_{n}:=\frac{1}{n} \sum_{l=1}^{n} \tilde{\beta}_{l} .
$$

\footnotetext{
${ }^{3}$ Note that the extra factor $(d-1) ! /\left(2 \pi^{d}\right)$ appearing in [Kra Thm. 1.5.5] is due to a different normalisation of the measure $\sigma$ on $\partial B_{1}$.
} 
To obtain (11) and (2) from (8) we require an upper bound on $\alpha_{n}$ and a lower bound on $\beta_{n}$. We start with the bounds for $\alpha_{n}$. Observe that

$$
\tilde{\alpha}_{1}=h_{d-1}(0)=1, \text { and } \tilde{\alpha}_{l} \leq d(l-1)^{1-1 / d} \text { for } l \geq 2 .
$$

To see this note that

$$
\frac{h_{d-1}(k)}{h_{d}(k-1)^{1-1 / d}}=\frac{(d !)^{1-1 / d}}{(d-1) !}\left(\frac{\prod_{l=1}^{d-1}(k+l)^{d}}{\prod_{l=0}^{d-1}(k+l)^{d-1}}\right)^{1 / d}=\frac{(d !)^{1-1 / d}}{(d-1) !} \prod_{l=1}^{d-1}\left(1+\frac{l}{k}\right)^{1 / d}
$$

is decreasing in $k$, so if $h_{d}(k-1)<n \leq h_{d}(k)$ then $\frac{\tilde{\alpha}_{l}}{(l-1)^{1-1 / d}} \leq \frac{h_{d-1}(k)}{h_{d}(k-1)^{1-1 / d}} \leq \frac{h_{d-1}(1)}{h_{d}(0)^{1-1 / d}}=d$.

The estimate (9) now yields the upper bound

$$
\alpha_{n}=\prod_{i=1}^{n} \tilde{\alpha}_{i}^{1 /(2 n)} \leq \sqrt{d}((n-1) !)^{(d-1) /(2 d n)} \leq \sqrt{d}\left(2\left(\frac{n}{e}\right)^{n}\right)^{(d-1) /(2 d n)} \leq \sqrt{d} n^{(d-1) /(2 d)},
$$

where, for $n>1$, we have used the estimate $(n-1) ! \leq 2\left(\frac{n}{e}\right)^{n}$ (i.e. $\log (n-1) ! \leq \int_{x=2}^{n} \log x d x \leq$ $n \log n-n+\log 2$ ).

We now turn to the bounds for $\beta_{n}$. If $h_{d}(k-1)<l \leq h_{d}(k)$, so that $\tilde{\beta}_{l}=k$, then $l \leq h_{d}(k) \leq(d !)^{-1}(k+d)^{d}$, which implies $\tilde{\beta}_{l}=k \geq(d !)^{1 / d} l^{1 / d}-d$. Therefore

$$
\beta_{n}=\frac{1}{n} \sum_{l=1}^{n} \tilde{\beta}_{l} \geq-d+(d !)^{1 / d} \frac{1}{n} \sum_{l=1}^{n} l^{1 / d}>-d+(d !)^{1 / d} \frac{d}{d+1} n^{1 / d},
$$

where we have used $\sum_{l=1}^{n} l^{1 / d}>\int_{x=0}^{n} x^{1 / d} d x=\frac{d}{d+1} n^{1+1 / d}$.

Assertion (11) now follows from (8), (10), and (11). Finally, if $d=1$ then $\beta_{n}=\frac{1}{n} \sum_{l=1}^{n} \tilde{\beta}_{l}=$ $\frac{1}{n} \sum_{l=1}^{n}(l-1)=(n-1) / 2$, and (10) becomes $\alpha_{n} \leq 1$, so substituting into (18) yields (2) .

\section{References}

[Bal] V. Baladi, Positive transfer operators and decay of correlations, Advanced series in nonlinear dynamics vol. 16, World Scientific, Singapore-New Jersey-London-Hong Kong, 2000.

[BJ] O. F. Bandtlow \& O. Jenkinson, Explicit eigenvalue estimates for transfer operators acting on spaces of holomorphic functions, Advances in Math., to appear.

[CCR] F. Christiansen, P. Cvitanović, \& H.-H. Rugh, The spectrum of the period-doubling operator in terms of cycles, J. Phys. A, 23 (1990), L713-L717.

[Dol] D. Dolgopyat, On decay of correlations in Anosov flows, Ann. Math., 147 (1998), 357-390.

[FR] F. Faure \& N. Roy, Ruelle-Pollicott resonances for real analytic hyperbolic maps, Nonlinearity, 19 (2006), 1233-1252.

[Fri] D. Fried, Zeta functions of Ruelle and Selberg I, Ann. Sci. Ec. Norm. Sup., 9 (1986) 491-517.

[GLZ] L. Guillopé, K. Lin \& M. Zworski, The Selberg zeta function for convex co-compact Schottky groups, Comm. Math. Phys., 245 (2004), 149-176.

[Hal] P. R. Halmos, A Hilbert space problem book, Springer-Verlag, New York-Heidelberg-Berlin, 1967.

[JMS] Y. Jiang, T. Morita \& D. Sullivan, Expanding direction of the period-doubling operator, Comm. Math. Phys., 144 (1992), 509-520.

[Kra] S. G. Krantz, Function theory of several complex variables, 2nd edition, AMS Chelsea, 1992.

[May] D. H. Mayer, On the thermodynamic formalism for the Gauss map, Comm. Math. Phys., 130 (1990), 311-333.

[Nar] R. Narasimhan, Several complex variables, University of Chicago Press, Chicago, 1971.

[Pie] A. Pietsch, Eigenvalues and s-numbers, CUP, Cambridge, 1987.

[Pol] M. Pollicott, A note on the Artuso-Aurell-Cvitanovic approach to the Feigenbaum tangent operator, J. Stat. Phys., 62 (1991), 257-267.

[PS] M. Pollicott \& R. Sharp, Correlations for pairs of closed geodesics, Invent. Math., 163 (2006), 1-24.

[Rud] W. Rudin, Function theory in the unit ball of $C^{n}$, Grundlehren der Mathematischen Wissenschaften 241, Springer-Verlag, New York-Berlin, 1980.

[Rue1] D. Ruelle, Statistical mechanics of a one-dimensional lattice gas, Comm. Math. Phys., 9 (1968), 267-278.

[Rue2] D. Ruelle, Zeta-functions for expanding maps and Anosov flows, Invent. Math., 34 (1976), 231-242 
[Wey] H. Weyl, Inequalities between two kinds of eigenvalues of a linear transformation, Proc. Nat. Acad. Sci. USA, 35 (1949), 408-411.

Oscar F. Bandtlow; School of Mathematical Sciences, Queen Mary, University of London, Mile End Road, London, E1 4NS, UK.

ob@maths . qmul.ac.uk

www. maths. qmul.ac.uk/ ob

Oliver Jenkinson; School of Mathematical Sciences, Queen Mary, University of London, Mile End RoAd, London, E1 4NS, UK.

omj@maths . qmul . ac . uk

www. maths.qmul.ac.uk/ omj 